\title{
Molecular Clouds in the Magellanic System
}

\author{
Yi-nan Chin \\ Academia Sinica Institute of Astronomy \& Astrophysics, PO Box 1-87 \\ Nankang, 11529 Taipei, Taiwan
}

\begin{abstract}
Several molecular cores in the Magellanic Clouds have been investigated. While previous works only concentrated on "warm" clouds, our study provides the first systematic study to cover different environments. Preliminary results are presented with brief discussions.
\end{abstract}

\section{Introduction}

Temperature, density, metallicity, and radiation field are important parameters to characterize the physical and chemical state of molecular clouds. Observationally, it is possible to vary the first two parameters (temperature and density) within our Milky Way by observing clouds in different locations. The metallicity, however, does not change drastically in the plane of the Milky Way. As two of the closest galaxies, the Magellanic Clouds provide metallicities which are factors of 3 and 10 lower (Westerlund 1991). If we treat our Galaxy as a "chemically" evolved system, the Magellanic System are without doubt still in an early stage of "chemical" evolution, with elemental abundances that may resemble those characterizing larger galaxies at high redshifts. In addition, the radiation field is stronger than in the solar neighborhood. As a consequence of the low metallicities and strong UV radiation field, the Magellanic Clouds are characterized by low dust-to-gas mass ratios. They are thus a "laboratory" where we can study molecular clouds with varied boundary conditions and it is easy to foresee that detailed observations will have a great impact on our general knowledge of astrochemistry and astrophysics of interstellar clouds.

\section{Observations}

The observations were carried out during several periods between 1996 and 1998 using the 15-m Swedish-ESO Submillimetre Telescope (SEST) at La Silla, Chile. A prominent molecular cloud in each of the Magellanic Clouds has been observed. Nevertheless, this work as well as the previous study (e.g., Johansson et al. 1994; Chin et al. 1997, 1998) have so far concentrated only on molecular cores associated with prominent $\mathrm{H}$ II regions. This does not sample the entire range of physical and chemical properties. Extending our studies to dark clouds would introduce a second molecular environment and would make a systematic comparison between the Magellanic system and our own Galaxy more meaningful. Recently, a hot interstellar region with enhanced X-ray emission in the LMC has been detected by $R O S A T$ and showed a large X-ray shadow to its western side. 
No H II emission was seen toward this shadow. This result encourages us to look for dense molecular cores in this area. Diffuse CO gas with some clumps has been detected and one of these quiescent molecular cores has been investigated in detail.

\section{Discussion}

In Table 1 some selected line intensity ratios of observed molecules are listed toward two molecular cores associated with prominent $\mathrm{H}$ II regions (N113 in the LMC and LIRS36 in the SMC), as well as a quiescent molecular core (DC1) for the first time found in the LMC. Previous studies toward various Galactic molecular cores and extragalactic centers have also been presented in the table for comparison. There are several factors which can affect the molecular abundance. For instance, the under-abundance of nitrogen-bearing molecules characterizes an early phase of "chemical" evolution.

Table 1. Selected line intensity ratios of observed molecules ${ }^{\dagger}$

\begin{tabular}{lcccccc}
\hline \multirow{2}{*}{ Source } & $\mathrm{HCO}^{+}$ & $\mathrm{HCN}$ & $\mathrm{CN}$ & $\mathrm{HC}_{2}$ & $\mathrm{CS}$ & $\mathrm{CS}$ \\
\hline N113 & 1.51 & 2.56 & 1.16 & 0.51 & 0.85 & 0.73 \\
LIRS 36 & 3.39 & 2.87 & $<0.27$ & 2.25 & 2.07 & $>7.5$ \\
DC 1 & 1.68 & 2.18 & 0.38 & 0.89 & 1.44 & 3.77 \\
N159 $\ddagger$ & 2.21 & 2.97 & 0.48 & - & 0.84 & 1.75 \\
S 138 & 0.67 & 3.75 & - & - & - & - \\
M17SW & 0.16 & 1.9 & $\ll 1$ & - & - & - \\
IRC+10216 & - & 8.24 & 1.24 & - & 0.45 & 0.36 \\
IC 443 & 1.2 & 7.0 & 1.7 & - & 0.85 & 0.5 \\
W49N & 1.0 & 6.0 & 0.5 & $>25.0$ & 1.0 & 2.0 \\
NGC 253 & 0.81 & 1.3 & 1.5 & 0.20 & 0.77 & 0.51 \\
IC 342 & 0.51 & 3.3 & 0.79 & - & 0.25 & 0.32 \\
M 82 & 2.1 & 2.0 & 1.4 & 0.46 & 0.95 & 0.68 \\
NGC 4945 & 0.93 & 2.03 & 1.48 & 0.45 & 0.52 & 0.35 \\
\hline \hline
\end{tabular}

† All transitions are in the $3 \mathrm{~mm}$ range, i.e., the $J=1-0$ transitions for the listed molecules, except $J=2-1$ for CS.

$\ddagger$ Another molecular cloud near 30 Dor in the LMC, observed by Johansson et al. 1994 .

\section{References}

Chin, Y.-N., Henkel, C., Whiteoak, J.B., et al. 1997, A\&A, 317, 548

Chin, Y.-N., et al. 1998, A\&A, 330, 901

Johansson, L.E.B., Olofsson, H., Hjalmarson, A., Gredel, R., \& Black, J.H. 1994, A\&A, 291, 89

Westerlund, B.E. 1990, A\&AR, 2, 29 